\title{
Valley-enhanced fast relaxation of gate-controlled donor qubits in silicon
}

\author{
Péter Boross,${ }^{1}$ Gábor Széchenyi, ${ }^{1}$ and András Pályi ${ }^{2}$ \\ ${ }^{1}$ Institute of Physics, Eötvös University, Budapest, Hungary \\ ${ }^{2}$ Department of Physics and MTA-BME Condensed Matter Research Group, \\ Budapest University of Technology and Economics, Budapest, Hungary
}

(Dated: July 18, 2018)

\begin{abstract}
Gate control of donor electrons near interfaces is a generic ingredient of donor-based quantum computing. Here, we address the question: how is the phonon-assisted qubit relaxation time $T_{1}$ affected as the electron is shuttled between the donor and the interface? We focus on the example of the 'flip-flop qubit' [Tosi et al., arXiv:1509.08538v1], defined as a combination of the nuclear and electronic states of a phosphorous donor in silicon, promising fast electrical control and long dephasing times when the electron is halfway between the donor and the interface. We theoretically describe orbital relaxation, flip-flop relaxation, and electron spin relaxation. We estimate that the flip-flop qubit relaxation time can be of the order of $100 \mu \mathrm{s}, 8$ orders of magnitude shorter than the value for an on-donor electron in bulk silicon, and a few orders of magnitude shorter (longer) than the predicted inhomogeneous dephasing time (gate times). All three relaxation processes are boosted by (i) the nontrivial valley structure of the electron-phonon interaction, and (ii) the different valley compositions of the involved electronic states.
\end{abstract}

\section{INTRODUCTION}

Donor-based spin qubits in silicon ${ }^{1 / 3}(\mathrm{Si})$ are promising building blocks for quantum information processing schemes, mainly due to qubit lifetimes that are prolonged by the weakness of spin-orbit and hyperfine interactions in this material ${ }^{413}$ Recent important experimental achievements of the field include initialization, coherent control and readout of electronic and nuclear spins of individual phosphorous $(\mathrm{P})$ donors,, $11|14| 15$ as well as increasing qubit lifetimes ${ }^{9|10| 12|16| 17}$ by using isotopically purified samples with strongly increased abundance of the nuclear-spin-free $\mathrm{Si}-28$ isotope.

A ubiquitous ingredient of donor-based quantuminformation processing schemes is to use electrical gates to control the wave function of the donor electron (see Fig. 1a). That often means that the electron is shuttled between the donor and a nearby interface $\frac{118 \sqrt{27}}{21}$ For example, in the Kane proposal ${ }^{1}$ gate control is suggested to tune the hyperfine interaction strength and to allow for exchange-based two-qubit operations. Here, we address the following question: how does the phononassisted qubit relaxation time $T_{1}$ depend on the location of the electron, as it is placed in an intermediate position between the donor and the interface? We focus on the example of the recently proposed ${ }^{25}$ flip-flop qubit (see Fig. 11F); it is defined as a combination of the nuclear and electronic states of a phosphorous donor in silicon, and it is expected to allow for fast electrical control and long dephasing times, when the gate-induced electric fields locate the electron halfway between the donor and the interface.

\section{A. Flip-flop qubit}

Naturally, most of the coherent-control experiments with donor-based spin qubits are performed using ac a)

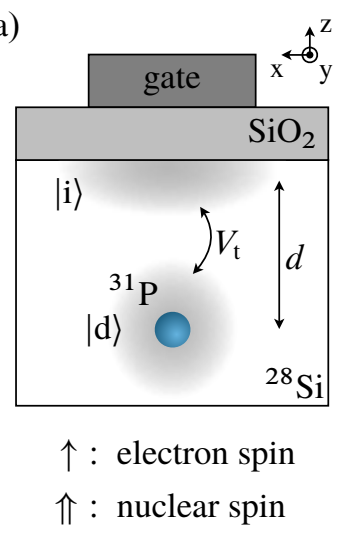

b)



FIG. 1. (Color online) Flip-flop qubit: setup and relaxation processes. (a) A donor is placed in the vicinity of a $\mathrm{Si} / \mathrm{SiO}_{2}$ interface, so that its electron can be moved between the interface $(|\mathrm{i}\rangle)$ and the donor $(|\mathrm{d}\rangle)$ by the voltage applied to the gate electrode. $V_{\mathrm{t}}$ : tunnel amplitude between $|\mathrm{i}\rangle$ and $|\mathrm{d}\rangle ; d$ : distance between the charge centers associated to $|\mathrm{i}\rangle$ and $|\mathrm{d}\rangle$. (b) Charge qubit basis states and orbital relaxation $\left(T_{1,0}\right)$ at the ionization point, $\epsilon_{\mathrm{o}}=V_{\mathrm{t}}$. (c) Energy diagram showing the combined states of the electronic and nuclear spins of the donor. The flip-flop qubit basis states are highlighted as thick black lines, their energy splitting is $\epsilon_{\mathrm{ff}}$. Flip-flop relaxation $\left(T_{1, \mathrm{ff}}\right)$ and electron spin relaxation $\left(T_{1, \mathrm{~s}}\right)$ both lead to information loss.

magnetic fields in the spirit of paramagnetic resonance. However, for a number of practical reasons, it can be advantageous to substitute the magnetic excitation with electrical driving, which is possible if a sufficiently strong interaction exists between the spin qubit and electric fields. On a single-qubit level, such an interaction allows local control via ac gate-voltage pulses, $28 \mid 30$ and dispersive non-demolition readout via probing a nearby electromagnetic resonator ${ }^{31}$ It also 
enables two-qubit operations, either via electric dipoledipole interaction, $25|29| 32 \mid 33]$ or via an electromagnetic resonator that mediates interaction between the qubits 31 These two-qubit gates, in contrast to the exchangebased gate, should be robust against donor placement uncertainties. 113435

The flip-flop qubit ${ }^{25}$ is expected to interact strongly with electric fields, and therefore has the potential to realize the desired features outlined above. The qubit is encoded in the composite system of the electronic and nuclear spins of a $\mathrm{P}$ donor, such that the qubit basis states are given by the two anti-aligned spin configurations $\uparrow \Downarrow$ and $\downarrow \Uparrow$, where the first (second) arrow represents the electronic (nuclear) spin. Importantly, the flip-flop terms of the hyperfine interaction between the electronic and nuclear spins couple the two qubit basis states. As a consequence, an ac electric field can drive coherent Rabi oscillations of the qubit: the field shakes the electronic wave function, thereby modulates the hyperfine coupling strength, which is in turn felt by the qubit as an ac Hamiltonian matrix element that couples the basis states.

This interaction between the flip-flop qubit and electric fields can be strongly enhanced in the configuration shown in Fig. 17. Here, the donor is placed in the vicinity of an interface between silicon and a barrier material (e.g., $\mathrm{SiO}_{2}$ ). If the charge center of the electron is approximately halfway between the donor ion and the interface (ionization point), then the coupling between the qubit and electric fields is maximized. A further advantage of such a setting is the existence of dephasing sweet spots in the space of the control parameters, including second-order clock-transition points where both the first and second derivatives of the qubit's Larmor frequency with respect to the dc electric field are zero. Tuning the system to such a sweet spot might result in exceptionally strong resilience against electrically-induced dephasing.

\section{B. This work}

In this work, we theoretically describe phononmediated relaxation of the flip-flop qubit, and determine the corresponding relaxation time $T_{1, \mathrm{ff}}$ (see Fig. $1 \mathrm{c}$ and section IV]. Reference 36 estimated a very long lowtemperature relaxation time of $T_{1, \mathrm{ff}} \sim 10^{4} \mathrm{~s}$ for a $\mathrm{P}$ donor in bulk silicon, set by deformation-induced changes of the effective mass and the dielectric constant. (Phononmediated spin relaxation processes involving nuclear-spin ensembles are treated, e.g., in Refs. 37 39.) In contrast, here we describe a deformation-potential mechanism that is particularly strong in the proposed working point of the flip-flop qubit, when the electron is at the ionization point, and leads to a characteristic $T_{1, \mathrm{ff}} \sim 100 \mu \mathrm{s}$. This time scale is approximately 8 orders of magnitude shorter than the prediction of Ref. 36, and a few orders of magnitude shorter (longer) than the predicted ${ }^{25}$ inhomogeneous dephasing time (gate times) of the flip-flop qubit.
The reason for the relatively fast relaxation is twofold. First, the flip-flop qubit is designed to strongly interact with electric fields at its working point, and that is achieved via hyperfine-induced mixing of the groundstate orbital with a low-lying excited orbital ${ }^{25}(|\mathrm{~g}\rangle$ and $|\mathrm{e}\rangle$, to be introduced below). The same low-lying excited orbital also provides strong interaction between the flip-flop qubit and phonon-induced deformation potentials, leading to relatively fast qubit relaxation. Second, we show that the relaxation process is valley-enhanced, where valley refers to the 6 conduction-band minima of the electronic band structure of silicon. In particular, the relaxation is boosted by the nontrivial valley-related features of the electron-phonon interaction and the involved electronic states.

We also characterize orbital relaxation, that is, relaxation of the charge qubit $\left(T_{1, o}\right.$ in Fig. $\left.1 \mathrm{~b}\right)$. Since orbital relaxation is conceptually simpler than the flip-flop relaxation, we start with the case of orbital relaxation in section III, and use it to introduce and illustrate the key ingredients of the valley-enhanced mechanism that governs all the three processes we consider (orbital, flip-flop, and electron spin relaxation). Finally, in section V, we describe electron spin relaxation ${ }^{4 / 11 \mid 13}$ from the excited state of the flip-flop qubit $\left(T_{1, \mathrm{~s}}\right.$ in Fig. 1 f): this process is also relevant for the functionality of the flip-flop qubit, as it leads to leakage from the qubit subspace.

\section{THE FLIP-FLOP QUBIT AND ITS MODEL HAMILTONIAN}

Here, based on Ref. 25, we discuss the setup in which the flip-flop qubit is envisioned, a simple 8-dimensional model Hamiltonian that captures the essential ingredients of the setup, and the 2-dimensional flip-flop qubit subspace. We note that in Ref. 25, this 8-dimensional Hamiltonian was found to reliably reproduce various physical quantities obtained from atomistic tight-binding simulations. This fact promotes this model to a trustable starting point for exploring the relaxation mechanisms of the flip-flop qubit.

In the absence of gate-induced electric fields, the donor electron is localized at the donor site, occupying the ground-state donor orbital $|\mathrm{d}\rangle$ (see Fig. 1 1). A voltage applied on the gate electrode induces an electric field along the $\mathrm{z}$ axis, and hence can pull the electron to the vicinity of the silicon-barrier interface, where it occupies the orbital state $|\mathrm{i}\rangle$. This two-orbital charge qubit degree of freedom is described by the Pauli matrices $\sigma_{\mathrm{x}}$, $\sigma_{\mathrm{y}}, \sigma_{\mathrm{z}}$, where, e.g., $\sigma_{\mathrm{z}}=|\mathrm{i}\rangle\langle\mathrm{i}|-| \mathrm{d}\rangle\langle\mathrm{d}|$. By continuously changing the gate-induced electric field $E_{\mathrm{z}}$, the electron is continuously moved between the two localized orbitals; the corresponding Hamiltonian reads

$$
H_{\mathrm{o}}=\frac{V_{\mathrm{t}}}{2} \sigma_{\mathrm{x}}-\frac{e\left(E_{\mathrm{z}}-E_{\mathrm{z}}^{0}\right) d}{2} \sigma_{\mathrm{z}},
$$

where $V_{\mathrm{t}}$ is the tunnel amplitude between the orbital states $|\mathrm{i}\rangle$ and $|\mathrm{d}\rangle$, and $E_{\mathrm{z}}^{0}$ is the value of the gate-induced 
electric field along $\mathrm{z}$ where the stationary electron charge is equally distributed among $|\mathrm{i}\rangle$ and $|\mathrm{d}\rangle$. The splitting between the energy eigenvalues of $H_{\mathrm{o}}$ is

$$
\epsilon_{\mathrm{o}}=\sqrt{V_{\mathrm{t}}^{2}+\left[e\left(E_{\mathrm{z}}-E_{\mathrm{z}}^{0}\right) d\right]^{2}} .
$$

An external homogeneous magnetic field introduces Zeeman splittings for both the electron and the nuclear spin of the donor. For simplicity, for the moment we assume isotropic and location-independent $g$-tensors, yielding the following electronic and nuclear Zeeman Hamiltonians, respectively:

$$
\begin{aligned}
& H_{B, e}=h \gamma_{\mathrm{e}} \boldsymbol{B} \boldsymbol{S}, \\
& H_{B, n}=h \gamma_{\mathrm{n}} \boldsymbol{B} \boldsymbol{I} .
\end{aligned}
$$

If the electron is located on the donor, then its spin $\boldsymbol{S}$ interacts with the nuclear spin $\boldsymbol{I}$ of the donor. Hence the hyperfine interaction is described by the following Hamiltonian:

$$
H_{\mathrm{hf}}=A\left(\frac{1-\sigma_{\mathrm{z}}}{2}\right) \boldsymbol{S I} .
$$

Here, both $\boldsymbol{S}$ and $\boldsymbol{I}$ are represented by $1 / 2$ times the vector of Pauli matrices. We introduce the secular $H_{\mathrm{hf}, \mathrm{sec}}$ and non-secular or flip-flop part $H_{\mathrm{hf}, \mathrm{ff}}=H_{\mathrm{hf}}-H_{\mathrm{hf}, \mathrm{sec}}$ of the hyperfine Hamiltonian, where the former is defined as

$$
H_{\mathrm{hf}, \mathrm{sec}}=A\left(\frac{1-\sigma_{\mathrm{z}}}{2}\right)\left(\boldsymbol{S} \cdot \frac{\boldsymbol{B}}{B}\right)\left(\boldsymbol{I} \cdot \frac{\boldsymbol{B}}{B}\right) .
$$

That is, $H_{\mathrm{hf}, \mathrm{sec}}$ incorporates spin components that are parallel to the external magnetic field, whereas $H_{\mathrm{hf}, \mathrm{ff}}$ incorporates the flip-flop terms.

The energy eigenstates of the $8 \times 8$ Hamiltonian $H_{\text {sec }}=$ $H_{\mathrm{o}}+H_{B, n}+H_{B, e}+H_{\mathrm{hf}, \mathrm{sec}}$ are direct products of the energy eigenstates $|\mathrm{g}\rangle$ and $|\mathrm{e}\rangle$ of $H_{\mathrm{o}}$ and electron $(\uparrow, \downarrow)$ and nuclear $(\Uparrow, \Downarrow)$ spin states pointing along the external magnetic field. These states will be labelled by the above quantum numbers and denoted as, e.g., $|\mathrm{g} \downarrow \uparrow\rangle_{0}$, and we will call them the unperturbed energy eigenstates.

If the spectral gaps of $H_{\mathrm{sec}}$ are much larger than the energy scale $A / 4$ characterizing $H_{\mathrm{hf}, \mathrm{ff}}$, then the latter remains a perturbation, and the energy eigenstates of the full Hamiltonian $H=H_{\mathrm{sec}}+H_{\mathrm{hf}, \mathrm{ff}}$ are approximately direct products as above, hence can be labelled with the same quantum numbers, and will be denoted as, e.g., $|\mathrm{g} \downarrow \uparrow\rangle$. Using this notation, the basis states of the flipflop qubit are $|\mathrm{g} \downarrow \uparrow\rangle$ and $|\mathrm{g} \uparrow \downarrow\rangle$. An example parameter set where the above conditions are met, and which was studied extensively in Ref. 25, is shown in Table [1. The level diagram consisting of the four energy eigenstates of $H$ associated to the ground-state orbital manifold is depicted in Fig. 17; there, the flip-flop qubit basis states, having an energy separation of $\epsilon_{\mathrm{ff}} \approx h \gamma_{\mathrm{e}} B$, are highlighted as bold black lines.
TABLE I. Parameter values. Remarks: (1) Working-point parameters are taken from Ref. 25, except the value of $E_{\mathrm{z}}-E_{\mathrm{z}}^{0}$, which we set to zero for simplicity. (2) Having no estimate for the spin-dependent tunnel matrix element $V_{\mathrm{s}}$, the quoted value is an arbitrary choice.

\begin{tabular}{lcc} 
Parameter & Notation & Value \\
\hline \hline Material-specific parameters & & \\
\hline Mass density of silicon & $\rho$ & $2330 \mathrm{~kg} / \mathrm{m}^{3}$ \\
Uniaxial deformation potential & $\Xi_{u}$ & $8.77 \mathrm{eV}$ \\
Longitudinal sound velocity & $v_{\mathrm{L}}$ & $9330 \mathrm{~m} / \mathrm{s}$ \\
Transverse sound velocity & $v_{\mathrm{T}}$ & $5420 \mathrm{~m} / \mathrm{s}$ \\
Hyperfine interaction strength for Si:P & $A / h$ & $117 \mathrm{MHz}$ \\
Gyromagnetic ratio of electron spin & $\gamma_{\mathrm{e}}$ & $27.97 \mathrm{GHz} / \mathrm{T}$ \\
Gyromagnetic ratio of P nuclear spin & $\gamma_{\mathrm{n}}$ & $17.23 \mathrm{MHz} / \mathrm{T}$ \\
\hline Working-point parameters & \multicolumn{2}{c}{} \\
\hline Magnetic field & $B$ & $0.2 \mathrm{~T}$ \\
Electric-field detuning from ionizaton point & $E_{\mathrm{z}}-E_{\mathrm{z}}^{0}$ & $0 \mathrm{~V} / \mathrm{m}$ \\
Donor-interface hopping amplitude & $V_{\mathrm{t}} / h$ & $5.91 \mathrm{GHz}$ \\
Donor-interface center-of-charge distance & $d$ & $15 \mathrm{~nm}$ \\
\hline Further parameters & \multicolumn{2}{c}{} \\
\hline Spin-dependent tunnel matrix element & $V_{\mathrm{s}} / h$ & $10 \mathrm{MHz}$ \\
Perpendicular g-tensor anisotropy & $\Delta_{\gamma}^{\perp}$ & $-0.2 \%$ \\
Parallel g-tensor anisotropy & $\Delta_{\gamma}^{\|}$ & $0.7 \%$ \\
Amplitude of ac electric field & $E_{\mathrm{ac}}$ & $32 \mathrm{~V} / \mathrm{m}$
\end{tabular}

\section{ORBITAL RELAXATION}

First, we characterize the phonon-emission-mediated orbital relaxation, that is, relaxation from the excited state $|\mathrm{e}\rangle$ of the charge qubit to its ground state $|\mathrm{g}\rangle$, and calculate the corresponding relaxation time $T_{1, \mathrm{o}}$, see Fig. 11p. We disregard the spin degrees of freedom for simplicity; the charge qubit is described by the $2 \times 2$ Hamiltonian $H_{\mathrm{o}}$ of Eq. (1), and the eigenstates $|\mathrm{g}\rangle$ and $|\mathrm{e}\rangle$ of $H_{\mathrm{o}}$ are called the charge qubit basis states. The valley-enhanced, deformation-potential-induced relaxation mechanism we describe here, as well as the structure of the calculation itself, is easily translated to treat the flip-flop relaxation and electron spin relaxation processes, which will be discussed in the subsequent sections.

\section{A. Preliminaries}

To account for the phonons and the electron-phonon interaction, we use a bulk-type description, neglecting any effects arising from inhomogeneities in the nanostructure.

In the experimentally relevant range of parameters, the charge-qubit energy splitting is resonant with low-energy long-wavelength acoustic phonons. Hence only those are considered here. Their dispersion relations are assumed to be linear and characterized by the sound velocities $v_{\lambda}$, where $\lambda \in(\mathrm{L}, \mathrm{T} 1, \mathrm{~T} 2)$ is the polarization index and $\mathrm{L}(\mathrm{T})$ refers to longitudinal (transverse).

We focus on the case of zero temperature and use the corresponding Fermi's Golden Rule to evaluate the qubit 
relaxation time:

$$
\frac{1}{T_{1, \mathrm{o}}}=\frac{2 \pi}{\hbar} \sum_{\boldsymbol{q}, \lambda}\left|\left\langle\mathrm{g}, \boldsymbol{q} \lambda\left|H_{\mathrm{eph}}\right| \mathrm{e}, 0\right\rangle\right|^{2} \delta\left(\epsilon_{\mathrm{o}}-\hbar v_{\lambda} q\right) .
$$

Here, bras and kets represent joint states of the composite electron-phonon system, 0 denotes the vacuum of phonons, and $\boldsymbol{q}(\lambda)$ is the wave number (polarization index) of the emitted phonon.

The mechanism we describe is based on the deformation-potential electron-phonon interaction, which we treat via the silicon-specific Herring-Vogt Hamiltonian! 4142

$$
H_{\mathrm{eph}}=\Xi_{u}\left(\begin{array}{cccccc}
\varepsilon_{x x} & 0 & 0 & 0 & 0 & 0 \\
0 & \varepsilon_{x x} & 0 & 0 & 0 & 0 \\
0 & 0 & \varepsilon_{y y} & 0 & 0 & 0 \\
0 & 0 & 0 & \varepsilon_{y y} & 0 & 0 \\
0 & 0 & 0 & 0 & \varepsilon_{z z} & 0 \\
0 & 0 & 0 & 0 & 0 & \varepsilon_{z z}
\end{array}\right) \text {, }
$$

where the $6 \times 6$ matrix structure corresponds to valley space, that is, the 6 envelope functions associated to the 6 conduction-band valleys of silicon, denoted and ordered as $(x, \bar{x}, y, \bar{y}, z, \bar{z})$. In Eq. (8), $\Xi_{u}$ is the uniaxial deformation potential and $\varepsilon$ is the strain tensor. Note that in addition to the right hand side of Eq. (8), the HerringVogt Hamiltonian incorporates a conventional, valleyindependent deformation-potential term, $\Xi_{d} \operatorname{Tr}(\varepsilon) 1_{6 \times 6}$, where $\Xi_{d}$ is the dilational deformation potential, $\operatorname{Tr}(\varepsilon)$ is the deformation-induced relative volume change, and $1_{6 \times 6}$ is the $6 \times 6$ unit matrix; however, we disregard that term here as (i) it does not contribute to the valleyenhanced mechanism to be described here, and (ii) its contributions to the relaxation rates obtained here are much smaller than those of the uniaxial deformation potential term.

The diagonal elements of the strain tensor, that is, the elements that determine $H_{\mathrm{eph}}$ via Eq. (8), read

$$
\varepsilon_{j j}=i \sqrt{\frac{\hbar}{2 \rho V}} \sum_{\boldsymbol{q}, \lambda} \frac{e_{\boldsymbol{q} \lambda j} q_{j}}{\sqrt{v_{\lambda} q}} e^{i \boldsymbol{q} \cdot \boldsymbol{r}}\left(a_{\boldsymbol{q}, \lambda}+a_{-\boldsymbol{q}, \lambda}^{\dagger}\right) .
$$

Here, $j \in\{\mathrm{x}, \mathrm{y}, \mathrm{z}\}, \rho$ is the mass density of silicon, $V$ is the sample volume and $\boldsymbol{e}_{\boldsymbol{q} \lambda}$ is the polarization vector of the phonon mode with wave number $\boldsymbol{q}$ and polarization index $\lambda$. For the setup we consider, the wavelength of the phonon emitted by the qubit is much longer than the spatial size of the qubit itself. Therefore, the plane-wave factor in Eq. (9) can be approximated as

$$
e^{i \boldsymbol{q} \cdot \boldsymbol{r}} \approx 1
$$

this corresponds to a homogeneous deformation, and as we will show, such a homogeneous deformation is sufficient to induce the described relaxation processes.

To obtain $T_{1, \mathrm{o}}$ via Fermi's Golden Rule (7), we need to provide the envelope-function representation of the localized charge states $|i\rangle$ and $|d\rangle$. For the purpose of obtaining the order of magnitude and the parameter dependence of the relaxation rates, it is sufficient to use simple 'perfectly localized' envelope functions, dressed by

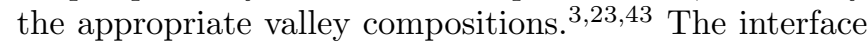
state $|i\rangle$ resembles that of a planar quantum-dot ground state pushed toward the barrier by the gate-induced electric field, hence its wave function resides in the $z$ and $\bar{z}$ valleys, evenly distributed. The donor state $|d\rangle$, on the other hand, is evenly distributed in all the 6 valleys. Using these considerations, we represent the two localized charge states as

$$
\begin{aligned}
\langle\boldsymbol{r} \mid \mathrm{i}\rangle & =\sqrt{\delta\left(\boldsymbol{r}-\boldsymbol{r}_{\mathrm{i}}\right)} \frac{1}{\sqrt{2}}\left(0,0,0,0, e^{i \phi_{z}}, e^{i \phi_{\bar{z}}}\right), \\
\langle\boldsymbol{r} \mid \mathrm{d}\rangle & =\sqrt{\delta(\boldsymbol{r})} \frac{1}{\sqrt{6}}(1,1,1,1,1,1),
\end{aligned}
$$

where $\delta(\boldsymbol{r})$ is the three-dimensional Dirac delta, the donor position is chosen as the origin of the reference frame, $\boldsymbol{r}_{\mathrm{i}}$ is the center of charge of the orbital $|\mathrm{i}\rangle$, and the phases $\phi_{z}$ and $\phi_{\bar{z}}$ are between 0 and $2 \pi$, but their actual values turn out to be irrelevant. In Eq. (11), the Dirac delta is a strongly simplified representation of the envelope functions associated to the valleys. We emphasize that a more realistic representation, e.g., using KohnLuttinger ${ }^{43}$ envelope functions for the donor orbital $|\mathrm{d}\rangle$, would only lead to minor quantitative corrections of our results.

Before evaluating the orbital relaxation time, it is instructive to restrict the electron-phonon interaction Hamiltonian $H_{\text {eph }}$ to the charge-qubit Hilbert space:

$$
H_{\mathrm{eph}, \mathrm{o}}=P H_{\mathrm{eph}} P=\Xi_{u} \frac{i}{6} \sqrt{\frac{\hbar}{2 \rho V}} \Sigma_{\mathrm{z}} \sigma_{\mathrm{z}},
$$

where $P=|\mathrm{i}\rangle\langle\mathrm{i}|+| \mathrm{d}\rangle\langle\mathrm{d}|$,

$$
\Sigma_{\mathrm{z}}=\sum_{\boldsymbol{q}, \lambda} \frac{\left(-e_{\boldsymbol{q} \lambda x} q_{x}-e_{\boldsymbol{q} \lambda y} q_{y}+2 e_{\boldsymbol{q} \lambda z} q_{z}\right)}{\sqrt{v_{\lambda} q}}\left(a_{\boldsymbol{q}, \lambda}+a_{-\boldsymbol{q}, \lambda}^{\dagger}\right) .
$$

Here we used Eqs. (8), (9), (10) and (11), and from Eq. (12) we omitted an irrelevant term proportional to the unit matrix $\sigma_{0}$. Remarkably, $H_{\mathrm{eph}, \mathrm{o}}$ is proportional to $\sigma_{\mathrm{z}}$, which means that there is a deformation-induced potential difference between the interface and donor sites, in spite of the homogeneous nature of the considered deformation component. The appearance of that effective potential difference is due to two factors: the nontrivial valley structure of the Herring-Vogt Hamiltonian, see Eq. 8), and the different valley compositions of the two localized orbitals $|i\rangle$ and $|d\rangle$, see Eq. (11).

Let us illustrate that claim, and the corresponding physical mechanism, with a simple example. Take a longitudinal phonon propagating along the $\mathrm{x}$ axis. This case corresponds to $\epsilon_{y y}=\epsilon_{z z}=0$ and a finite $\epsilon_{x x}$. Hence, according to Eq. (8), the conduction-band edges in the $x$ and $\bar{x}$ valleys are raised by the uniaxial deformation potential $\Xi_{u} \epsilon_{x x}$, whereas the conduction band edges in the other four valleys are not affected. Then, this effective potential in the $x$ and $\bar{x}$ valleys is felt differently 
by $|\mathrm{i}\rangle$ and $|\mathrm{d}\rangle$ : the state $|\mathrm{i}\rangle$ has no weight in the $x$ and $\bar{x}$ valleys [see Eq. [11a]], therefore it does not feel the presence of the deformation; the state $|\mathrm{d}\rangle$, however, has a total weight of $1 / 3$ in the $x$ and $\bar{x}$ valleys together [see Eq. [11b]], and hence the deformation raises its potential energy by $\Xi_{u} \epsilon_{x x} / 3$. Therefore we conclude that a homogeneous deformation indeed induces a potential energy difference between the interface orbital and the donor orbital. Furthermore, our argument translates to an effective electron-phonon coupling Hamiltonian with a nontrivial part of $-\Xi_{u} \epsilon_{x x} \sigma_{z} / 6$, in line with the corresponding term in Eq. 12.

\section{B. Results}

To obtain the orbital relaxation time, Fermi's Golden Rule (7) is evaluated as

$$
\frac{1}{T_{1, \mathrm{o}}}=\frac{\epsilon_{\mathrm{o}} V_{t}^{2} \Xi_{u}^{2}}{60 \pi \hbar^{4} \rho}\left(\frac{2}{3 v_{\mathrm{L}}^{5}}+\frac{1}{v_{\mathrm{T}}^{5}}\right),
$$

where we used

$$
\left\langle\mathrm{g}\left|\sigma_{z}\right| \mathrm{e}\right\rangle=V_{t} / \epsilon_{\mathrm{o}} .
$$

At the ionization point, where $\epsilon_{\mathrm{o}}=V_{t}$, and using the working-point parameters specified in Table $\mathbb{I}$ the orbital relaxation rate is estimated as $1 / T_{1, \mathrm{o}} \approx 0.49 \mathrm{MHz}$, corresponding to a relaxation time of $T_{1, \mathrm{o}} \approx 2.1 \mu \mathrm{s}$.

Upon detuning from the ionization point, the charge qubit energy splitting $\epsilon_{\mathrm{o}}$ increases, and therefore, according to Eq. 14, the relaxation speeds up. This is interpreted as the result of a competition between three effects.

First, relaxation should slow down upon detuning from the ionization point because the charge qubit basis states $|g\rangle$ and $|e\rangle$ become more localized, which suppresses the relevant matrix element $\left\langle\mathrm{g}\left|\sigma_{z}\right| \mathrm{e}\right\rangle$. Second, relaxation should be enhanced upon detuning from the ionization point, as the charge qubit energy splitting $\epsilon_{\mathrm{o}}$ increases, and therefore the density of states of the available phonons also increases. These two mechanisms exactly cancel each other.

The fact that the relaxation speeds up upon detuning from the ionization point is therefore a consequence of a third fact: the vacuum fluctuation of the strain of a phonon mode with energy $\epsilon_{\mathrm{o}}$ is proportional to $\sqrt{\epsilon_{\mathrm{o}}}$; that follows from Eqs. (9) and (10), and the energy conservation condition $\epsilon_{\mathrm{o}}=\hbar v_{\lambda} q$ embedded in Fermi's Golden Rule (7). The quadratic form of Fermi's Golden Rule then implies a $1 / T_{1, \mathrm{o}} \propto \epsilon_{\mathrm{o}}$ dependence due to this factor, which does indeed appear in our result (14).

\section{Valley-enhanced relaxation}

We wish to highlight the fact that the nontrivial features of the setup associated to the valley degree of freedom boost the orbital relaxation process, and will play the same role in the flip-flop relaxation and electron spin relaxation processes to be described below. In that sense, all these can be considered valley-enhanced relaxation processes. Our argument supporting that claim is as follows. The two relevant features are (i) the nontrivial valley structure of the electron-phonon interaction, and (ii) the different valley compositions of the localized charge states $|i\rangle$ and $|d\rangle$. In the absence of any of these two ingredients, the first, homogeneous-deformation term in the plane-wave expansion $e^{i \boldsymbol{q} \cdot \boldsymbol{r}} \approx 1+i \boldsymbol{q} \cdot \boldsymbol{r}+\ldots$ of the strain tensor (9) would give a vanishing contribution to the relaxation rate, and therefore the relaxation rate would be suppressed by a factor of $(q d)^{2}$. Using the parameter values of Table $\mathbb{I}$, that factor has the value of $(q d)^{2} \approx 3 \times 10^{-3}\left[(q d)^{2} \approx 10^{-2}\right]$ for longitudinal [transverse] phonons.

In conclusion, in this section we have described a phonon-emission-mediated orbital relaxation process, characteristic of a charge qubit formed by a gate-tuned electron located between its donor atom and a nearby interface. In particular, we have shown that the relaxation process is enhanced by (i) the nontrivial valley structure of the electron-phonon interaction and (ii) the different valley compositions of the two orbital wave functions forming the charge qubit.

\section{FLIP-FLOP RELAXATION}

Here, we use the model described in sections II and III to characterize the phonon-emission-mediated relaxation process from the flip-flop qubit excited state $\mid \mathrm{g} \downarrow \uparrow \uparrow$ to its ground state $|\mathrm{g} \uparrow \Downarrow\rangle$. This process is labelled in Fig. 1p as $T_{1, \mathrm{ff}}$. The characteristic time scale of this process for an isolated $\mathrm{P}$ donor at low temperature in bulk silicon has been estimated ${ }^{36}$ as $T_{1, \mathrm{ff}} \sim 10^{4} \mathrm{~s}$. Here we show that this time scale can decrease by approximately 8 orders of magnitudes, that is, $T_{1, \mathrm{ff}} \sim 100 \mu \mathrm{s}$ is possible, if the flipflop qubit is tuned to couple strongly to electric fields.

The flip-flop relaxation mechanism is visualized using the level diagram in Fig. 2a. It can be thought of as a twostep or second-order process, in which matrix elements of the flip-flop part of the hyperfine interaction $H_{\mathrm{hf}, \mathrm{ff}}$, depicted as solid arrows in Fig.22a, and matrix elements of the electron-phonon interaction $H_{\mathrm{eph}}$, denoted as dashed arrows in Fig. 2 a, provide relaxation paths via virtual intermediate states.

Our calculation of $T_{1, \mathrm{ff}}$ follows the preliminaries and derivation steps of the calculation of $T_{1, \mathrm{o}}$ in the previous section. For the flip-flop relaxation rate, Fermi's Golden Rule reads

$$
\frac{1}{T_{1, \mathrm{ff}}}=\frac{2 \pi}{\hbar} \sum_{\boldsymbol{q}, \lambda}\left|\left\langle\mathrm{g} \downarrow \uparrow, \boldsymbol{q} \lambda\left|H_{\mathrm{eph}, \mathrm{o}}\right| \mathrm{g} \uparrow \Downarrow, 0\right\rangle\right|^{2} \delta\left(\epsilon_{\mathrm{ff}}-\hbar v_{\lambda} q\right) .
$$

As long as $H_{\mathrm{hf}, \mathrm{ff}}$ is a perturbation of $H_{\mathrm{sec}}$, we can use first-order perturbation theory to obtain analytical approximate expressions for the qubit basis states $|\mathrm{g} \downarrow \uparrow \uparrow\rangle$ 




b)



c)

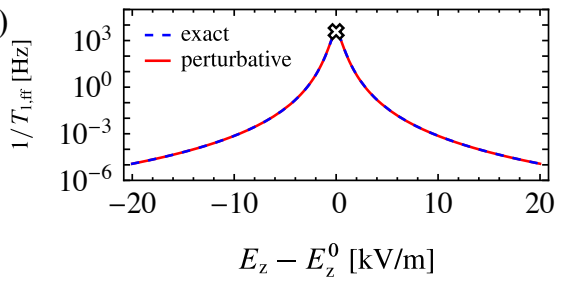

d)

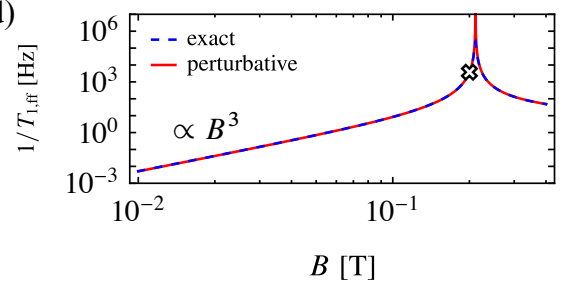

FIG. 2. (Color online) Flip-flop relaxation via spontaneous phonon emission. (a) Hyperfine flip-flop matrix elements (solid arrows) and electron-phonon matrix elements (dashed arrows) enabling flip-flop relaxation. (b) Flip-flop relaxation rate $1 / T_{1, \mathrm{ff}}$ as a function of electric and magnetic fields. The white cross corresponds to the working point in Table I further parameter values are also specified there. See text for definition of the flip-flop qubit basis states. (c,d) Blue dashed: horizontal/vertical cut of (b) through the working point (white cross in b,c,d). Red solid: analytical perturbative result, Eq. 17).

and $|\mathrm{g} \uparrow \Downarrow\rangle$ in terms of the 8 unperturbed energy eigenstates. In fact, the form of $H_{\mathrm{hf}, \mathrm{ff}}$ guarantees that the flip-flop qubit basis states are linear combinations of the 4 unperturbed energy eigenstates $|\mathrm{g} \downarrow \uparrow\rangle_{0},|\mathrm{~g} \uparrow \downarrow\rangle_{0},|\mathrm{e} \downarrow \uparrow\rangle_{0}$, and $|\mathrm{e} \uparrow \Downarrow\rangle_{0}$. The flip-flop relaxation rate is then readily evaluated from Eq. (16) as:

$$
\frac{1}{T_{1, \mathrm{ff}}}=\frac{A^{2} \Xi_{u}^{2} V_{\mathrm{t}}^{4} \epsilon_{B}^{3}}{240 \pi \hbar^{4} \rho \epsilon_{\mathrm{o}}^{2}\left(\epsilon_{\mathrm{o}}^{2}-\epsilon_{B}^{2}\right)^{2}}\left(\frac{2}{3 v_{\mathrm{L}}^{5}}+\frac{1}{v_{\mathrm{T}}^{5}}\right) \text {. }
$$

Here, $\epsilon_{B}=h \gamma_{\mathrm{e}} B$, and Eq. 17 shows the leading-order result in the small parameters $A /\left(\epsilon_{\mathrm{o}}-\epsilon_{B}\right), h \gamma_{\mathrm{n}} B /\left(\epsilon_{\mathrm{o}}-\epsilon_{B}\right) \ll 1$.

This result can also be expressed in terms of the orbital relaxation time:

$$
\frac{1}{T_{1, \mathrm{ff}}}=\frac{1}{4} \frac{A^{2} V_{\mathrm{t}}^{2} \epsilon_{B}^{3}}{\epsilon_{\mathrm{o}}^{3}\left(\epsilon_{\mathrm{o}}^{2}-\epsilon_{B}^{2}\right)^{2}} \frac{1}{T_{1, \mathrm{o}}},
$$

taking a particularly simple approximate form in the vicinity of the proposed working point, where the electron is placed halfway between the interface and the donor and the energy splittings of the charge qubit and flipflop qubit are similar $\left(V_{\mathrm{t}} \approx \epsilon_{\mathrm{o}} \approx \epsilon_{B}\right)$ :

$$
\frac{1}{T_{1, \mathrm{ff}}} \approx\left(\frac{A / 4}{\epsilon_{\mathrm{o}}-\epsilon_{B}}\right)^{2} \frac{1}{T_{1, \mathrm{o}}} .
$$

Note that this result corresponds to the special case when the leftmost virtual transition of Fig. 2 dominates the relaxation process.

With the parameter values in Table I, from Eq. (17) we obtain $1 / T_{1, \mathrm{ff}} \approx 3.7 \mathrm{kHz}$, implying a flip-flop relaxation time of $T_{1, \mathrm{ff}} \approx 270 \mu \mathrm{s}$. This value is approximately 8 orders of magnitude shorter than the $10^{4} \mathrm{~s}$ time scale that was estimated for an on-donor electron in bulk by
Ref. 36. The reason for the fast relaxation at the proposed working point of the flip-flop qubit is is twofold. First, the working point is chosen with the goal of optimizing the speed of electrically driven qubit transitions: $\epsilon_{\mathrm{o}}-\epsilon_{B}$, which appears as an energy denominator in the perturbative description of the leftmost virtual process of Fig. 2, is chosen to be relatively small $(\sim h \times 300 \mathrm{MHz})$, so that the qubit excited state $|\mathrm{g} \uparrow \Downarrow\rangle$ has a relatively large, hyperfine-mediated admixture with the unperturbed energy eigenstate $|\mathrm{e} \downarrow \uparrow\rangle_{0}$. Second, this flip-flop relaxation process is valley-enhanced, in a similar sense as described in section III C. That is, due to the nontrivial valley structure of the electron-phonon Hamiltonian and the different valley compositions of the involved electronic orbitals $|i\rangle$ and $|\mathrm{d}\rangle$, even a uniform phonon-induced deformation is capable to induce relaxation.

If the charge-qubit splitting $\epsilon_{\mathrm{o}}$ is much larger than the electronic Zeeman splitting $\epsilon_{B}$, then Eq. (17) implies the power-law relation $1 / T_{1, f f} \propto B^{3}$, see also Fig. 2 d. The $3 r d$ power arises as a sum $1+2$, where the terms, respectively, are associated to the strain vacuum fluctuations and the density of states of three-dimensional acoustic phonons. This is analogous to the low-temperature limiting case $1 / T_{1, \mathrm{ff}} \propto B^{3}$ of the relaxation mechanism considered in Ref. 36 for on-donor electrons in bulk: even though the mechanisms considered here and there are different, in both cases a homogeneous deformation is responsible for the relaxation.

In Fig. 2 b, we show the dependence of the qubit relaxation rate $1 / T_{1, \mathrm{ff}}$ on the gate-induced electric field and the magnetic field. To obtain this result, we first numerically computed the eigenvalues and eigenvectors of $H$. Then, we identified the flip-flop qubit ground (excited) state as the energy eigenstate having the largest overlap with $|\mathrm{g} \downarrow \uparrow\rangle_{0}\left(|\mathrm{~g} \uparrow \downarrow\rangle_{0}\right)$. Finally, we evaluated the 
relaxation rate according to Eq. 16.

The key features in Fig. 2 $\mathrm{p}$ are as follows. (i) The qubit relaxation rate is strongly suppressed at low magnetic fields, due to the above-discussed $1 / T_{1, \mathrm{ff}} \propto B^{3}$ dependence. (ii) The qubit relaxation rate is maximal, taking values around $1 \mathrm{MHz}$, along the upward-bending hyperbola, which corresponds to $\epsilon_{\mathrm{o}} \approx \epsilon_{B}$, and, therefore, nonperturbative mixing of $|\mathrm{g} \uparrow \Downarrow\rangle$ and $|\mathrm{e} \downarrow \uparrow\rangle$. Hence this relaxation rate of $1 \mathrm{MHz}$ reflects the orbital relaxation rate.

Comparison of the numerical results of Fig. $2 \mathrm{~b}$ and the perturbative, analytical expression (17) is shown in Fig. 2k,d. In Fig. 2k, the dashed blue line shows a horizontal cut of Fig. $2 \mathrm{~b}$ through the working point (white cross), whereas the solid red line is the analytical result. A similar comparison, corresponding to a vertical cut of Fig. $2 \mathrm{~b}$ through the working point, is shown in Fig. $2 \mathrm{~b}$. Note that in Fig. 2d , for magnetic fields slightly higher than the working-point magnetic field, the analytical result deviates from the numerical one and diverges; that behavior is an artefact arising from the breakdown of first-order perturbation theory.

In conclusion, we have proposed a valley-enhanced relaxation mechanism of the flip-flop qubit, calculated its characteristic relaxation time $T_{1, \mathrm{ff}}$, and found a relatively short, $\sim 100 \mu$ s time scale in the proposed working point. This is partly due to the presence of a low-lying orbital that is utilized to enhance the coupling of the qubit to the electric field. Another factor boosting the relaxation process is the absence of dipole suppression (see section IIIC): thanks to the nontrivial valley structure of the electron-phonon interaction and the involved electronic orbitals $|i\rangle$ and $|d\rangle$, a homogeneous deformation can induce an effective potential difference between the two orbitals, and hence lead to efficient relaxation.

\section{ELECTRON SPIN RELAXATION}

A further process, leading to leakage from the flip-flop qubit subspace, is electron spin relaxation (henceforth spin relaxation, for short): this is shown in Fig. 1;, labelled as $T_{1, \mathrm{~s}}$. We first describe a valley-enhanced spinrelaxation mechanism that is enabled by spin-orbit interaction; more precisely, by spin-dependent electron tunnelling between the two localized orbitals $|\mathrm{i}\rangle$ and $|\mathrm{d}\rangle$. We also discuss an alternative valley-enhanced relaxation mechanism, which is enabled by the feature that the $g$ tensors characterizing the localized orbitals $|\mathrm{i}\rangle$ and $|\mathrm{d}\rangle$ are, in general, different and anisotropic. 40

\section{A. Spin relaxation due to spin-dependent tunneling}

First, we incorporate spin-orbit interaction to our $8 \times 8$ model Hamiltonian described in section II. For simplicity, we assume that the setup is cylindrically symmetric around the $\mathrm{z}$ axis. We claim that this symmetry condition, together with the condition that the spin-orbit Hamiltonian must be invariant under time reversal, imply the following simple form for the spin-orbit Hamiltonian:

$$
H_{\mathrm{so}}=V_{\mathrm{s}} \sigma_{\mathrm{y}} S_{\mathrm{z}}
$$

where $V_{\mathrm{s}}$ is real. Naturally, this Hamiltonian excludes the nuclear-spin operators. Furthermore, since $\sigma_{\mathrm{y}}$ is an offdiagonal matrix, $H_{\text {so }}$ describes spin-dependent tunneling between the two orbitals $|\mathrm{i}\rangle$ and $|\mathrm{d}\rangle$.

The proof of Eq. 200, inspired by a related argument of Ref. 44, is as follows. In principle, the spinorbit Hamiltonian can be expanded in terms of products of charge-qubit Pauli matrices including the unit matrix $\sigma_{0}$, and the three spin Pauli matrices: $H_{\mathrm{so}}=$ $\sum_{i=0, \mathrm{x}, \mathrm{y}, \mathrm{z}} \sum_{j=\mathrm{x}, \mathrm{y}, \mathrm{z}} \alpha_{i j} \sigma_{i} S_{j}$, where $\alpha_{i j}$ represent 12 unknown coefficients. Then, the condition of time reversal invariance renders 9 of the coefficients zero, $\alpha_{0 j}=$ $\alpha_{\mathrm{x} j}=\alpha_{\mathrm{z} j}=0$, for the following reason. Time reversal is represented as $T=2 i S_{\mathrm{y}} K$ with $K$ being the complex conjugation, therefore the spin matrices $S_{\mathrm{x}}, S_{\mathrm{y}}$, and $S_{\mathrm{z}}$ change sign under time reversal, whereas the real matrices $\sigma_{0}, \sigma_{\mathrm{x}}$ and $\sigma_{\mathrm{z}}$ keep their signs. That implies that the only charge-qubit Pauli matrix allowed in the spin-orbit Hamiltonian is $\sigma_{\mathrm{y}}: H_{\mathrm{so}}=\sigma_{\mathrm{y}}\left(\alpha_{\mathrm{yx}} S_{\mathrm{x}}+\alpha_{\mathrm{yy}} S_{\mathrm{y}}+\alpha_{\mathrm{yz}} S_{\mathrm{z}}\right)$. However, a finite value of either $\alpha_{\mathrm{yx}}$ or $\alpha_{\mathrm{yy}}$ would specify a certain direction in the xy plane, which is disallowed by the cylindrical symmetry of the setup around the $\mathrm{z}$ axis. With the identification $V_{\mathrm{s}} \equiv \alpha_{\mathrm{yz}}$, this concludes the proof of Eq. 200. A quantitative characterization of $V_{\mathrm{S}}$ could be obtained from microscopic, e.g., tight-binding, 40 simulations, incorporating the nanostructure geometry and spin-orbit interaction. Here, we treat $V_{\mathrm{s}}$ as a phenomenological parameter.

Having the spin-orbit Hamiltonian at hand, we now propose the spin relaxation mechanism it enables. The mechanism is analogous to the flip-flop relaxation, and is visualized using the level diagram in Fig. 33. Here, we parametrize the magnetic-field orientation via its polar angle $\theta: \quad \boldsymbol{B}=B(\sin \theta, 0, \cos \theta)$, but disregard any orbital effects caused by $\boldsymbol{B}$. Furthermore, recall that the arrows in our state notation (for example, in $|\mathrm{g} \uparrow \downarrow\rangle_{0}$ ) correspond to spin alignments with respect to the external magnetic field, not with respect to z. Then, we conclude that $H_{\text {so }}$ mixes the unperturbed state $|\mathrm{g} \uparrow \Downarrow\rangle_{0}$ with $|\mathrm{e} \downarrow \Downarrow\rangle_{0}$. This mixing is depicted as the left solid arrow in Fig. 3 a. Since, in turn, $|\mathrm{e} \downarrow \Downarrow\rangle_{0}$ is connected to $|\mathrm{g} \downarrow \Downarrow\rangle_{0}$ by the electron-phonon interaction (left dashed arrow in Fig. 3a), we conclude that the spin-orbit interaction does indeed enable spin relaxation. A similar two-step process contributing to spin relaxation is depicted by the right solid and dashed arrows.

The spin relaxation rate arising from these secondorder processes can be calculated as

$$
\frac{1}{T_{1, \mathrm{~s}}}=\frac{2 \pi}{\hbar} \sum_{\boldsymbol{q}, \lambda}\left|\left\langle\mathrm{g} \downarrow \Downarrow, \boldsymbol{q} \lambda\left|H_{\mathrm{eph}, \mathrm{o}}\right| \mathrm{g} \uparrow \Downarrow, 0\right\rangle\right|^{2} \delta\left(\epsilon_{\mathrm{s}}-\hbar v_{\lambda} q\right) .
$$



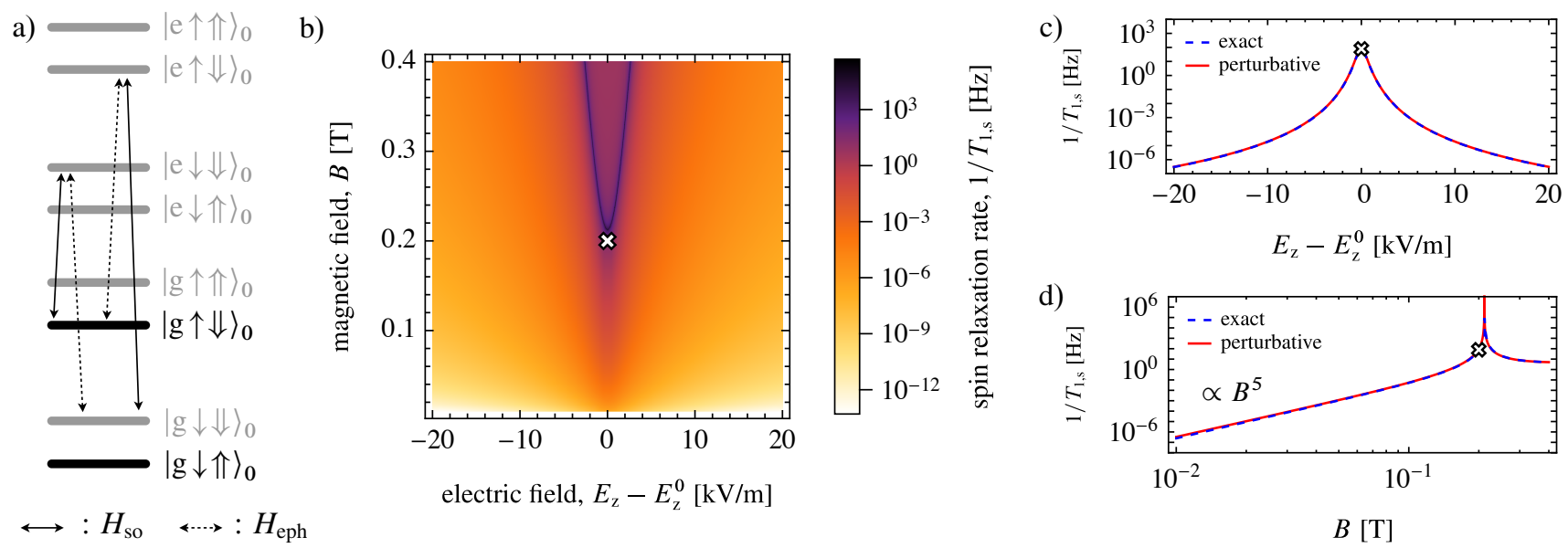

FIG. 3. (Color online) Leakage from the flip-flop qubit subspace: electron spin relaxation due to spin-dependent tunneling and spontaneous phonon emission. (a) Spin-dependent tunneling matrix elements (solid arrows) and electron-phonon matrix elements (dashed arrows) enabling electron-spin relaxation. (b) Electron spin relaxation rate $1 / T_{1, \mathrm{~s}}$ as a function of electric and magnetic fields, for $V_{\mathrm{s}} / h=10 \mathrm{MHz}$. The white cross corresponds to the working point in Table further parameter values are also specified there. (c,d) Blue dashed: horizontal/vertical cut of (b) through the working point (white cross). Red solid: analytical perturbative result, Eq. 22.

where the states $|g \downarrow \downarrow \downarrow\rangle$ and $|g \uparrow \Downarrow\rangle$ are perturbed by the spin-orbit interaction, in analogy to Eq. (16), where the states are perturbed by the flip-flop terms of the hyperfine interaction. Furthermore, $\epsilon_{s}$ is the energy splitting between the energy eigenstates $|\mathrm{g} \uparrow \Downarrow\rangle$ and $|\mathrm{g} \downarrow \Downarrow\rangle$.

Using first-order perturbation theory to account for the spin-orbit-induced mixing of the unperturbed states, we find

$$
\frac{1}{T_{1, \mathrm{~s}}}=\frac{V_{\mathrm{s}}^{2} \sin ^{2} \theta \Xi_{u}^{2} V_{\mathrm{t}}^{2} \epsilon_{B}^{5}}{15 \pi \hbar^{4} \rho \epsilon_{\mathrm{o}}^{2}\left(\epsilon_{\mathrm{o}}^{2}-\epsilon_{B}^{2}\right)^{2}}\left(\frac{2}{3 v_{\mathrm{L}}^{5}}+\frac{1}{v_{\mathrm{T}}^{5}}\right),
$$

which shows the leading-order result in the small parameters $A /\left(\epsilon_{\mathrm{o}}-\epsilon_{B}\right), h \gamma_{\mathrm{n}} B /\left(\epsilon_{\mathrm{o}}-\epsilon_{B}\right), V_{\mathrm{s}} /\left(\epsilon_{\mathrm{o}}-\epsilon_{B}\right) \ll 1$.

Expressed with the orbital relaxation rate:

$$
\frac{1}{T_{1, \mathrm{~s}}}=\frac{4 V_{\mathrm{s}}^{2} \sin ^{2} \theta \epsilon_{B}^{5}}{\epsilon_{\mathrm{o}}^{3}\left(\epsilon_{\mathrm{o}}^{2}-\epsilon_{B}^{2}\right)^{2}} \frac{1}{T_{1, \mathrm{o}}}
$$

In the vicinity of the proposed working point where $\epsilon_{\mathrm{o}} \approx$ $\epsilon_{B}$, this is approximated as

$$
\frac{1}{T_{1, \mathrm{~s}}} \approx\left(\frac{\frac{1}{2} V_{\mathrm{s}} \sin \theta}{\epsilon_{\mathrm{o}}-\epsilon_{B}}\right)^{2} \frac{1}{T_{1, \mathrm{o}}} .
$$

At weak magnetic fields, $\epsilon_{B} \ll \epsilon_{\mathrm{o}}$, the spin-relaxation rate in Eq. 22 follows the power-law relation $1 / T_{1, \mathrm{~s}} \propto$ $B^{5}$, see Fig. $3 \mathrm{~d}$; this is a stronger dependence then the $1 / T_{1, \text { ff }} \propto B^{3}$ seen in the previous section, and the difference is due to van Vleck cancellation!

In Fig. 3 b, we show the dependence of the spin relaxation rate $1 / T_{1, \mathrm{~s}}$ on the gate-induced electric field and the magnetic field, in analogy to Fig. 2, using the numerically computed eigenvalues and eigenvectors of $H+H_{\text {so }}$. To produce this plot, the magnetic field is assumed to be aligned with the $\mathrm{x}$ axis $(\theta=\pi / 2)$, and for not having a calculated or measured value for the spin-dependent tunneling energy $V_{\mathrm{s}}$, we used an arbitrary value $V_{\mathrm{s}} / h=10 \mathrm{MHz}$. That implies that even though the parameter dependencies of the spin relaxation rate shown in Fig. 3b,c,d are expected to be accurate, the actual numerical values should not be regarded as predictions. Having a realistic estimate $V_{\mathrm{s} \text {,est }}$ for the spindependent tunneling amplitude, the plotted results could be rescaled to provide numerical predictions by multiplying with $\left(V_{\mathrm{s}, \text { est }} / 10 \mathrm{MHz}\right)^{2}$.

The key features in Fig. $3 \mathrm{~b}$ are analogous to those of the flip-flop relaxation. (i) The spin relaxation rate is strongly suppressed at low magnetic fields, due to the above-discussed $1 / T_{1, \mathrm{~s}} \propto B^{5}$ dependence. (ii) The spin relaxation rate is maximal along an upward-bending hyperbola, corresponding to $\epsilon_{\mathrm{o}} \approx \epsilon_{B}$, and, therefore, nonperturbative mixing of $|g \uparrow \Downarrow\rangle$ and $|e \downarrow \Downarrow\rangle$. (iii) For the working point specified in Table I (white cross in Figs. $3 \mathrm{~b}, \mathrm{c}, \mathrm{d})$, the numerical value for the spin relaxation rate evaluated from Eq. 22 is $1 / T_{1, \mathrm{~s}} \approx 98 \mathrm{~Hz}$, that is, the spin relaxation time is $T_{1, \mathrm{~s}} \approx 10 \mathrm{~ms}$.

A comparison between the exact and perturbative results, for a horizontal (vertical) cut of Fig. $3 \mathrm{~b}$ across the working point, is shown in Figs. 3 c (d).

\section{B. Spin relaxation due to $g$-tensor modulation}

We conclude the list of valley-enhanced relaxation mechanisms with spin relaxation due to $g$-tensor modulation. This process is allowed if the $g$-tensors associated to the localized orbitals $|i\rangle$ and $|d\rangle$ are different, and, e.g., that of $|\mathrm{i}\rangle$ is anisotropic. ${ }^{[25 \mid 40]}$ In that case, a phonon, cor- 
responding to an effective potential difference between the two localized orbitals, redistributes the electron between the two locations and thereby changes the $g$-tensor. In general, that implies that both the length and the direction of the effective Zeeman field felt by the electron changes, leading to spin relaxation.

We focus on the simple case when the $g$-tensors show cylindrical symmetry along the growth direction z. Importantly, in this case, this relaxation process can be avoided by a perfect in-plane or out-of-plane alignment of the external magnetic field $\boldsymbol{B}$. We further assume that $g$-tensor anisotropy is present only at the interface. That anisotropy is incorporated in our model as the perturbation term

$$
H_{g \mathrm{tm}}=\left(\frac{1+\sigma_{\mathrm{z}}}{2}\right) h \gamma_{\mathrm{e}} \boldsymbol{B}\left(\begin{array}{ccc}
\Delta_{\gamma}^{\perp} & 0 & 0 \\
0 & \Delta_{\gamma}^{\perp} & 0 \\
0 & 0 & \Delta_{\gamma}^{\|}
\end{array}\right) \boldsymbol{S} .
$$

Tight-binding nanostructure models predict 40 that the typical absolute value of the relative $g$-tensor anisotropy parameters is in the range $\left|\Delta_{\gamma}^{\perp}\right|,\left|\Delta_{\gamma}^{\|}\right| \in[0.1,1] \%$.

To evaluate the corresponding spin relaxation time $T_{1, \mathrm{~s}}$, we follow the same procedure as in section $\mathrm{VA}$, but now instead of $H_{\text {so }}$, we use $H_{g \mathrm{tm}}$ as the perturbation in the Hamiltonian. (Note that the two mechanisms do interfere in general; we disregard that here and discuss their effects separately for simplicity.) The leading-order perturbative result, expressed using the orbital relaxation time $T_{1, \mathrm{o}}$, reads

$$
\frac{1}{T_{1, \mathrm{~s}}}=\frac{1}{16} \frac{\left[\left(\Delta_{\gamma}^{\perp}-\Delta_{\gamma}^{\|}\right) \sin (2 \theta)\right]^{2} V_{\mathrm{t}}^{2} \epsilon_{B}^{5}}{\epsilon_{\mathrm{o}}^{3}\left(\epsilon_{\mathrm{o}}^{2}-\epsilon_{B}^{2}\right)^{2}} \frac{1}{T_{1, \mathrm{o}}} .
$$

In the vicinity of the proposed working point where $V_{\mathrm{t}} \approx$ $\epsilon_{\mathrm{o}} \approx \epsilon_{B}$, this is approximated as

$$
\frac{1}{T_{1, \mathrm{~s}}} \approx\left[\frac{\epsilon_{B}\left(\Delta_{\gamma}^{\perp}-\Delta_{\gamma}^{\|}\right) \sin (2 \theta) / 8}{\epsilon_{\mathrm{o}}-\epsilon_{B}}\right]^{2} \frac{1}{T_{1, \mathrm{o}}} .
$$

With the parameters in Table I, we estimate the maximal spin relaxation rate, corresponding to a B-field polar angle of $\theta=\pi / 4$, as $1 / T_{1, \mathrm{~s}} \approx 140 \mathrm{~Hz}$, implying a spin relaxation time of $T_{1, \mathrm{~s}} \approx 7 \mathrm{~ms}$. Recall that under our presumptions, this mechanism can be fully suppressed by aligning the magnetic field in the xy plane or along the $\mathrm{z}$ axis; this appears explicitly in the results 26 and 27) via the factor $\sin (2 \theta)$.

In conclusion, we proposed that spin-orbit-induced spin-dependent tunneling between the localized charge states $|i\rangle$ and $|d\rangle$ can induce a valley-enhanced electron spin relaxation process, which leads to leakage from the flip-flop qubit subspace, and we expressed the parameter dependence of the corresponding relaxation rate. We also discussed how the different $g$-tensors characterizing the two localized charge states $|i\rangle$ and $|d\rangle$ can contribute to electron spin relaxation.

\section{DISCUSSION}

\section{A. Electrically driven spin resonance}

The spin relaxation process described in section $\mathrm{VA}$ is allowed by the spin-orbit-induced spin-dependent tunneling matrix element $V_{\mathrm{s}}$. The same matrix element could also be utilized for electrically driven electron spin resonance: an ac voltage component on the top gate produces an ac electric field $E_{\text {ac }}$ along z, which provides the same couplings as the electron-phonon matrix elements depicted as dashed arrows in Fig. 3 a, and thereby drives coherent transitions between $|\mathrm{g} \downarrow \Downarrow\rangle$ and $|\mathrm{g} \uparrow \Downarrow\rangle$. The corresponding Rabi frequency reads

$$
f_{\mathrm{s}, \text { Rabi }}=\frac{\epsilon_{B} V_{\mathrm{s}} V_{\mathrm{t}} \sin \theta e E_{\mathrm{ac}} d}{2 h \epsilon_{\mathrm{o}}\left(\epsilon_{\mathrm{o}}^{2}-\epsilon_{B}^{2}\right)} .
$$

This result is obtained via the following steps: we (i) expressed the energy eigenstates of the $8 \times 8$ model Hamiltonian $H_{\mathrm{sec}}+H_{\mathrm{hf}, \mathrm{ff}}+H_{\mathrm{so}}$ using first-order perturbation theory in $H_{\mathrm{hf}, \mathrm{ff}}+H_{\mathrm{so}}$, (ii) projected the driving Hamiltonian $H_{\mathrm{ac}}=e E_{\mathrm{ac}} z \sin (\omega t)$ onto the two-dimensional subspace spanned by the perturbed energy eigenstates $|\mathrm{g} \downarrow \Downarrow\rangle$ and $|\mathrm{g} \uparrow \Downarrow\rangle$, and (iii) read off the Rabi frequency as the amplitude of the transverse driving term in the resulting two-dimensional Hamiltonian. In the vicinity of the proposed working point, $V_{\mathrm{t}} \approx \epsilon_{\mathrm{o}} \approx \epsilon_{B}$, and in the presence of an in-plane magnetic field $(\theta=\pi / 2)$ the result 28 simplifies to

$$
f_{\mathrm{s}, \text { Rabi }}=\frac{V_{\mathrm{s}} e E_{\mathrm{ac}} d}{4 h\left(\epsilon_{\mathrm{o}}-\epsilon_{B}\right)} .
$$

Using the parameter values in Table I, and assuming an in-plane magnetic field $(\theta=\pi / 2)$, we find $f_{\mathrm{s}, \text { Rabi }} \approx$ $0.89 \mathrm{MHz}$.

For comparison, we provide the analogous result for the Rabi frequency of the electrically driven transitions of the flip-flop qubit:

$$
f_{\mathrm{ff}, \text { Rabi }}=\frac{A V_{\mathrm{t}}^{2} e E_{\mathrm{ac}} d}{4 h \epsilon_{\mathrm{o}}\left(\epsilon_{\mathrm{o}}^{2}-\epsilon_{B}^{2}\right)} .
$$

In the vicinity of the proposed working point, $V_{\mathrm{t}} \approx \epsilon_{\mathrm{O}} \approx$ $\epsilon_{B}$, the result $(30)$ simplifies to

$$
f_{\mathrm{ff}, \text { Rabi }}=\frac{A e E_{\mathrm{ac}} d}{8 h\left(\epsilon_{\mathrm{o}}-\epsilon_{B}\right)},
$$

and thereby we recover the corresponding result of Ref. 25 [2 times the coupling rate in Eq. (7) of Ref. 25].

Finally, we highlight a potential use of electrically driven spin resonance in the nuclear-spin-based quantum processor proposed in Ref. 25. For that setup, a key ingredient is a magnetic drive of the donor electron spin via an ac magnetic field. Creating such an ac magnetic field requires an extra element, for example, a microwave transmission line, in the setup. Electrically driven spin resonance, allowed by a sufficiently strong spin-dependent tunnel matrix element $V_{\mathrm{s}}$ and driven by 
an ac gate voltage component, could substitute the ac magnetic field, and hence reduce the complexity of the envisioned architecture. To assess the practical feasibility of electrically driven spin resonance, a quantitative characterization of $V_{\mathrm{s}}$ is required.

\section{B. Breaking of the approximate cylindrical symmetry affects spin relaxation}

In Ref. 25, it is proposed that the interface-donor tunneling amplitude $V_{\mathrm{t}}$ is tuned to the desired value by moving the interface orbital $|\mathrm{i}\rangle$ away from the donor orbital $|d\rangle$ along the interface, using an appropriately designed gate stack. Of course, in that case the approximate cylindrical symmetry assumed in our considerations of spin relaxation (section $\mathrm{V}$ ) is broken, and therefore our symmetry-based results have to be refined accordingly.

\section{Relaxation rates at finite temperature}

Here, we evaluated relaxation rates corresponding to zero temperature and spontaneous phonon emission. Induced-emission and absorption rates at finite temperature $T>0$ are obtained by multiplying the corresponding spontaneous-emission rates with the Bose-Einstein factor $n(\epsilon, T)=1 /\left(e^{\epsilon / k_{\mathrm{B}} T}-1\right)$, where $\epsilon$ is the energy of the involved phonons and $k_{\mathrm{B}}$ is the Boltzmann constant.

\section{Orbital relaxation: comparison to experiment}

A recent experiment ${ }^{26}$ reports an orbital relaxation time $T_{1, \mathrm{o}} \approx 0.1 \mu \mathrm{s}$ of a charge qubit, formed in an effective double quantum dot system in a silicon nanowire transistor, where one of the dots is presumably a single $\mathrm{P}$ donor, while the other one is gate-defined. The quoted orbital relaxation time, measured at the chargequbit anticrossing point at a nominal charge-qubit energy splitting of $\epsilon_{\mathrm{o}}=V_{\mathrm{t}}=h \times 5.5 \mathrm{GHz}$, can be compared to the corresponding prediction of our Eq. (14), that is $T_{1, \mathrm{o}} \approx 2.5 \mu \mathrm{s}$.

Note that even though the two setups, studied in Ref. 26 and in this work, share their hybrid dot-donor character, there are also important differences between them: (i) The charge qubit in the experiment is formed by two electrons, in the $(1,1)-(0,2)$ charge configuration, where the first (second) integer is the number of electrons in the quantum dot (on the donor); our result (14) corresponds to the single-electron case. (ii) In the experiment, the quantum dot is formed at the corner of a nanowire, which presumably implies that the valley composition of the occupied electronic state is different from that described by Eq. 11a), the latter corresponding to an electronic state at a (001) silicon/barrier interface. (iii) In the experiment, the inhomogeneous dephasing time of the charge qubit is comparable to its splitting at the $(1,1)$ $(0,2)$ anticrossing. This indicates the presence of relatively strong electrical noise affecting the charge qubit detuning or tunnel coupling. Therefore, the measured orbital relaxation time should probably be understood as an average over a random ensemble of the charge-qubit parameters.

Note also that the orbital relaxation time $T_{1, \mathrm{o}}$ was measured for a single setting of the charge qubit. Measuring $T_{1, \mathrm{o}}$ as a function of the charge-qubit parameters would allow a qualitative comparison with theoretically predicted trends, e.g., Eq. (14) of this work, and thereby help identifying the underlying relaxation mechanism.

\section{E. Prolonging the relaxation times}

(1) Controlling the valley composition of the donor orbital $|d\rangle$. In the valley-enhanced relaxation mechanisms described in this work, a key ingredient is the substantially different valley structure of the electronic wave functions at the interface and donor sites, see Eq. (11). Making the valley composition [Eq. (11b)] of the donor orbital more similar to that [Eq. 11a)] of the interface orbital would prolong the relaxation times. The even valley composition of $|d\rangle$ in Eq. 111b might be altered by a number of mechanisms: for example, by static strain due to a finite germanium concentration


interface, ${ }^{23 \mid 48}$ or by an electric field ${ }^{49}$ For example, placing the donor closer to an interface, while keeping all other relevant parameters unchanged, would bring the valley compositions of $|i\rangle$ and $|d\rangle$ closer to each other, and therefore presumably prolong the relaxation times considered here. That speculation is supported by, e.g., the estimate in Ref. 48, claiming that the z-valley population of a donor electron at $3.4 \mathrm{~nm}$ below a silicon surface is $\approx 40 \%$, in contrast to the bulk value $33 \%$.

(2) Optimizing the working point via weakening the qubit-field interaction. In the vicinity of the working point of Table I] the estimated ${ }^{25}$ time required for a cavity-mediated $\sqrt{\text { SWAP }}$ two-qubit gate is $\tau_{\sqrt{\text { SWAP }}} \approx$ $0.4 \mu \mathrm{s}$. This implies that the number of such operations performed during the flip-flop relaxation time is $T_{1, \mathrm{ff}} / \tau_{\sqrt{\mathrm{SWAP}}} \approx 680$. In principle, this quality factor can be improved via, e.g., increasing the tunneling amplitude $V_{\mathrm{t}}$, thereby weakening the hyperfine-induced hybridization of $|\mathrm{g} \uparrow \Downarrow\rangle_{0}$ with $|\mathrm{e} \downarrow \uparrow\rangle_{0}$ (see Fig. 2a), and hence weakening the interaction between the flip-flop qubit and the electric fields. For example, approximately a factor of 2 improvement of the above quality factor can be achieved by the following adjustments. (i) The tunnel matrix element is reset to $V_{\mathrm{t}}=6.2 \mathrm{GHz}$. Essentially, this doubles the energy denominator $\epsilon_{\mathrm{o}}-\epsilon_{B}$ in the flip-flop relaxation rate 19 as well as in the vacuum Rabi frequency; the latter is obtained from (31) by identifying $E_{\mathrm{ac}}$ with the cavity vacuum field. As a result, $T_{1, f f}$ increases by a factor of 4. (ii) The magnetic field is reset such that the 
TABLE II. Time scales at the working point of Table I Remarks: (1) Having no estimate for the spin-dependent tunnel matrix element $V_{\mathrm{s}}$, the values quoted below for 'Spin relaxation (spin-orbit)' and '1-qubit gate (spin, $\pi / 2$ )' should not be regarded as predictions. (2) Relaxation refers to phononemission-mediated relaxation. (3) The $\pi / 2$ single-qubit gate time is $1 / 4$ times the inverse Rabi frequency. (4) 'On-donor flip-flop relaxation in bulk' is calculated assuming $1.2 \mathrm{~K}$ and $h \times 9 \mathrm{GHz}$ flip-flop splitting.

\begin{tabular}{lcc} 
Processes/gates & Rate & Time \\
\hline \hline Flip-flop qubit gate times & & \\
\hline 1-qubit gate $(\pi / 2)$ & $22 \mathrm{MHz}$ & $45 \mathrm{~ns}$ \\
2-qubit gate $(\sqrt{\mathrm{SWAP}}$, cavity-mediated) & $2.5 \mathrm{MHz}$ & $400 \mathrm{~ns}$ \\
2-qubit gate $(\sqrt{\mathrm{SWAP}}$, dipole-dipole $\sqrt{25}$ & $25 \mathrm{MHz}$ & $40 \mathrm{~ns}$ \\
\hline Information loss of flip-flop qubit & & \\
\hline Flip-flop relaxation & $3.7 \mathrm{kHz}$ & $270 \mu \mathrm{s}$ \\
Spin relaxation (spin-orbit, $\theta=\pi / 2)$ & $98 \mathrm{~Hz}$ & $10 \mathrm{~ms}$ \\
Spin relaxation $(g$-tensor mod., $\theta=\pi / 4)$ & $140 \mathrm{~Hz}$ & $7 \mathrm{~ms}$ \\
Electrically-induced dephasing $\sqrt{25}$ & {$[1,300] \mathrm{Hz}$} & {$[3.3 \mathrm{~ms}, 1 \mathrm{~s}]$} \\
\hline Further time scales & & \\
\hline On-donor flip-flop relaxation in bulk $\sqrt{36}$ & $2.9 \times 10^{-5} \mathrm{~Hz}$ & $3.4 \times 10^{4} \mathrm{~s}$ \\
Orbital relaxation & $490 \mathrm{kHz}$ & $2.1 \mu \mathrm{s}$ \\
1-qubit gate (spin, $\pi / 2)$ & $3.6 \mathrm{MHz}$ & $280 \mathrm{~ns}$
\end{tabular}

qubit-cavity detuning is halved. As a result of (i) and (ii), $\tau_{\sqrt{\text { SWAP }}}$ increases only with a factor of 2 , without a significant change in the gate fidelity; hence the quality factor $T_{1, \mathrm{ff}} / \tau_{\sqrt{\mathrm{SWAP}}}$ indeed doubles. In practice, an important consideration that should be added to the above procedure is the change of the inhomogeneous dephasing time $T_{2, \mathrm{ff}}^{*}$ of the flip-flop qubit with the adjustments, with the goal of exploiting the expected long $T_{2, \mathrm{ff}}^{*}$ times offered by the second-order clock transitions. ${ }^{25}$ In general, this necessitates a more complex optimization procedure.

\section{CONCLUSIONS}

We described fast, valley-enhanced relaxation mechanisms (orbital, flip-flop and electron spin relaxation) for a gate-controlled $\mathrm{P}$ donor electron close to a silicon/barrier interface. For the flip-flop qubit setup and the proposed qubit working point, we have found that the flip-flop relaxation can be approximately 8 orders of magnitude faster than in bulk. The predicted relaxation time scale is $\sim 100 \mu \mathrm{s}$, still longer than the expected single-qubit $(40 \mathrm{~ns})$ and two-qubit (40 - $400 \mathrm{~ns})$ gate times. ${ }^{25} \mathrm{Nev}$ ertheless, relaxation might dominate dephasing, if our estimates as well as the inhomogeneous dephasing rate estimate $^{25} 1 / T_{2, \mathrm{ff}}^{*} \sim[1,300] \mathrm{Hz}$ are reliable. The relevant time scales are listed in Table $\amalg$.

We also discussed analogous, valley-enhanced mechanisms inducing orbital and electron spin relaxation. Since gate control of donor electrons near interfaces is an ubiquitous ingredient of donor-based quantumcomputing schemes, the relevance of the mechanisms described here extends beyond the considered specific flipflop qubit architecture.

\section{ACKNOWLEDGMENTS}

We thank D. Culcer, R. Joynt, and R. Rahman for useful discussions, and M. Calderón, A. Morello, and G. Tosi for their helpful and constructive feedback on the manuscript. We acknowledge funding from the EU Marie Curie Career Integration Grant CIG-293834, Hungarian OTKA Grants No. PD 100373 and 108676, the Gordon Godfrey Bequest, and the EU ERC Starting Grant 258789. A. P. was supported by the János Bolyai Scholarship of the Hungarian Academy of Sciences.
1 B. E. Kane, Nature 393, 133 (1998).

2 J. Morton, D. McCamey, M. Eriksson, and S. Lyon, Nature 479, 345 (2011).

${ }^{3}$ F. A. Zwanenburg, A. S. Dzurak, A. Morello, M. Simmons, L. Hollenberg, G. Klimeck, S. Rogge, S. Coppersmith, and M. Eriksson, Rev. Mod. Phys. 85, 961 (2013).

${ }^{4}$ G. Feher, Phys. Rev. 114, 1219 (1959).

${ }^{5}$ G. Feher and E. A. Gere, Phys. Rev. 114, 1245 (1959).

${ }^{6}$ L. M. Roth, Phys. Rev. 118, 1534 (1960).

7 H. Hasegawa, Phys. Rev. 118, 1523 (1960).

8 C. Tahan, M. Friesen, and R. Joynt, Phys. Rev. B 66, 035314 (2002)

${ }^{9}$ A. M. Tyryshkin, S. A. Lyon, A. V. Astashkin, and A. M. Raitsimring, Phys. Rev. B 68, 193207 (2003).

10 A. M. Tyryshkin, J. J. L. Morton, S. C. Benjamin, A. Ardavan, G. A. D. Briggs, J. W. Ager, and S. A. Lyon, J. Phys. Condens. Matter 18, S783 (2006).

11 A. Morello, J. J. Pla, F. A. Zwanenburg, K. W. Chan, H. Huebl, M. Mottonen, C. D. Nugroho, C. Yang, J. A. van Donkelaar, A. D. C. Alves, D. N. Jamieson, C. C. Escott, L. C. L. Hollenberg, R. G. Clark, and A. S. Dzurak, Nature 467, 687 (2010).
12 A. M. Tyryshkin, S. Tojo, J. J. L. Morton, H. Riemann, N. V. Abrosimov, P. Becker, H.-J. Pohl, T. Schenkel, M. L. W. Thewalt, K. M. Itoh, and S. A. Lyon, Nat Mater 11, 143 (2012).

13 C. Tahan and R. Joynt, Phys. Rev. B 89, 075302 (2014).

14 J. J. Pla, K. Y. Tan, J. P. Dehollain, W. H. Lim, J. J. L. Morton, D. N. Jamieson, A. S. Dzurak, and A. Morello, Nature 489, 541 (2012).

15 J. J. Pla, K. Y. Tan, J. P. Dehollain, W. H. Lim, J. J. L. Morton, F. A. Zwanenburg, D. N. Jamieson, A. S. Dzurak, and A. Morello, Nature 496, 334 (2013).

16 J. Muhonen, J. Dehollain, A. Laucht, F. Hudson, T. Sekiguchi, K. Itoh, D. Jamieson, J. McCallum, A. Dzurak, and A. Morello, Nature Nanotechnology 9, 986 (2014).

17 K. M. Itoh and H. Watanabe, MRS Communications 4, 143 (2014).

18 R. Vrijen, E. Yablonovitch, K. Wang, H. W. Jiang, A. Balandin, V. Roychowdhury, T. Mor, and D. DiVincenzo, Phys. Rev. A 62, 012306 (2000).

19 M. J. Calderón, B. Koiller, X. Hu, and S. Das Sarma, Phys. Rev. Lett. 96, 096802 (2006). 
20 M. J. Calderón, B. Koiller, and S. Das Sarma, Phys. Rev. B 77, 155302 (2008).

${ }^{21}$ G. P. Lansbergen, R. Rahman, C. J. Wellard, I. Woo, J. Caro, N. Collaert, S. Biesemans, G. Klimeck, L. C. L. Hollenberg, and S. Rogge, Nat Phys 4, 656 (2008).

${ }^{22}$ R. Rahman, G. P. Lansbergen, S. H. Park, J. Verduijn, G. Klimeck, S. Rogge, and L. C. L. Hollenberg, Phys. Rev. B 80, 165314 (2009)

23 A. Baena, A. L. Saraiva, B. Koiller, and M. J. Calderón, Phys. Rev. B 86, 035317 (2012).

24 A. Laucht, J. T. Muhonen, F. A. Mohiyaddin, R. Kalra, J. P. Dehollain, S. Freer, F. E. Hudson, M. Veldhorst, R. Rahman, G. Klimeck, K. M. Itoh, D. N. Jamieson, J. C. McCallum, A. S. Dzurak, and A. Morello, Science Advances 1 (2015), 10.1126/sciadv.1500022

${ }^{20}$ G. Tosi, F. A. Mohiyaddin, S. B. Tenberg, R. Rahman, G. Klimeck, and A. Morello, ArXiv:1509.08538v1.

${ }^{26}$ M. Urdampilleta, A. Chatterjee, C. C. Lo, T. Kobayashi, J. Mansir, S. Barraud, A. C. Betz, S. Rogge, M. F. Gonzalez-Zalba, and J. J. L. Morton, Phys. Rev. X 5, 031024 (2015)

27 P. Harvey-Collard, N. T. Jacobson, M. Rudolph, J. Dominguez, G. A. T. Eyck, J. R. Wendt, T. Pluym, J. K. Gamble, M. P. Lilly, M. Pioro-Ladrière, and M. S. Carroll, ArXiv:1512.01606v1.

28 V. N. Golovach, M. Borhani, and D. Loss, Phys. Rev. B 74, 165319 (2006).

${ }^{29}$ C. Flindt, A. S. Sørensen, and K. Flensberg, Phys. Rev. Lett. 97, 240501 (2006)

so K. C. Nowack, F. H. L. Koppens, Y. V. Nazarov, and L. M. K. Vandersypen, Science 318, 1430 (2007).

31 A. Blais, R.-S. Huang, A. Wallraff, S. M. Girvin, and R. J.
Schoelkopf, Phys. Rev. A 69, 062320 (2004)

32 M. Trif, V. N. Golovach, and D. Loss, Phys. Rev. B 75, 085307 (2007)

33 J. Salfi, J. A. Mol, D. Culcer, and S. Rogge, arXiv:1508.04259 (2015).

34 P. R. Cullis and J. R. Marko, Phys. Rev. B 1, 632 (1970)

35 B. Koiller, X. Hu, and S. Das Sarma, Phys. Rev. Lett. 88, 027903 (2001).

36 D. Pines, J. Bardeen, and C. P. Slichter, Phys. Rev. 106, 489 (1957)

37 A. Abragam, The principles of nuclear magnetism (Oxford University Press, 1961).

38 A. V. Khaetskii, Physica E 10, 27 (2001).

39 S. I. Erlingsson and Y. V. Nazarov, Phys. Rev. B 66, 155327 (2002).

40 R. Rahman, S. H. Park, T. B. Boykin, G. Klimeck, S. Rogge, and L. C. L. Hollenberg, Phys. Rev. B 80, 155301 (2009)

${ }^{41}$ C. Herring and E. Vogt, Phys. Rev. 101, 944 (1956).

42 P. Y. Yu and M. Cardona, Fundamentals of Semiconductors (Springer, Berlin, 2010).

43 W. Kohn and J. M. Luttinger, Phys. Rev. 98, 915 (1955)

44 J. Danon and Y. V. Nazarov, Phys. Rev. B 80, 041301 (2009)

45 J. H. van Vleck, Phys. Rev. 57, 426 (1940).

46 A. Khaetskii and Y. Nazarov, Phys. Rev. B 64, 125316 (2001).

47 B. Koiller, X. Hu, and S. Das Sarma, Phys. Rev. B 66, 115201 (2002).

48 J. Salfi, J. A. Mol, R. Rahman, G. Klimeck, M. Y. Simmons, L. C. L. Hollenberg, and S. Rogge, Nat. Mater. 13, 605 (2014).

49 M. Friesen, Phys. Rev. Lett. 94, 186403 (2005) 